\title{
Calorimetry and thermodynamic aspects of heterotrophic, mixotrophic, and phototrophic growth
}

\author{
Urs von Stockar • Ian Marison • Marcel Janssen • \\ Rodrigo Patiño
}

ISBCXVI Special Issue

(C) Akadémiai Kiadó, Budapest, Hungary 2011

\begin{abstract}
A simple stoichiometric model is proposed linking the biomass yield to the enthalpy and Gibbs energy changes in chemo-heterotrophic, mixotrophic, and photoautotrophic microbial growth. A comparison with calorimetric experiments on the algae Chlorella vulgaris and Chlorella sorokiniana confirmed the trends but revealed large calorimetric measurement inaccuracies. The calorimetric data on purely photo-autotrophic growth was, however, in fair agreement with calculations. The thermodynamic characteristics of photosynthetic growth, including an estimation of the Gibbs energy dissipation, are compared with similar data for chemotrophic microbes.
\end{abstract}

Keywords Bio-photo calorimetry - Reaction calorimetry · Photosynthetic growth · Mixotrophic growth · Clorella vulgaris . Biological dissipation of Gibbs energy

U. von Stockar $(\bowtie)$

Swiss Federal Institute of Technology (EPFL), ISIC, Station 6, 1015 Lausanne, Switzerland

e-mail: urs.vonstockar@epfl.ch

U. von Stockar · I. Marison

School of Biotechnology, Dublin City University (DCU),

Dublin 9, Ireland

M. Janssen

Department of Agrotechnology and Food Sciences,

Wageningen University, Wageningen, The Netherlands

R. Patiño

Departemento de Fisica Aplicada, CINVESTAV—Unitad

Mérida, Aparto Postal 73, Cordemex, 97310 Mérida,

Youcatan, Mexico

\section{Introduction}

Dwindling fossil resources on our planet and seriously disturbing accumulation of greenhouse gases in our atmosphere have initiated efforts to develop novel technologies that are expected to provide food, energy and raw materials in a more sustainable way and that would be much more based on biological processes. Both harnessing sunlight by biological processes or by other systems close to photosynthesis, as well as transforming molecules in one another by using fermentative or synthetic capabilities of microbial, animal, or plant cells, will be important components of these technologies. In developing these, a clear understanding of the energy exchanges and of the thermodynamic characteristics of such biological processes will be an important issue. Calorimetry is a convenient experimental tool to explore these characteristics, because it permits measuring experimentally one of these energy exchanges, namely heat dissipation.

Heat generation accompanying growth of chemoheterotrophic microorganism and other cells have been extensively studied [1-8]. Aerobic growth has been found highly exothermic in all cases. A similar statement may be made for anaerobic oxidative and fermentative growth, with the exception of the fact that the latter shows clearly weaker heat effects. Simple stoichiometric models have been designed to predict the amount of heat generated. Only one single type of culture, namely acetotrophic methanogenesis by the bacterial strain Methanosarcina barkeri, has been demonstrated calorimetrically to not grow exothermically, but endothermically [9]. In contrast to the vast majority of chemoheterotrophic growth photosynthesis would be expected to be endothermic because it represents the inverse of the aerobic oxidation of biomass, which is highly 
exothermic. This has indeed been confirmed by a number of calorimetric studies [10-15].

Patiño et al. [14] also reported calorimetric results on mixotrophic growth of the algae Chlorella vulgaris. These results are of a particular interest because the strain could be grown in a pure chemo-heterotrophic, a pure photoautotrophic and in mixotrophic modes involving various mixes of heterotrophic and autotrophic metabolism. Since simple formal predictions of the heat release and the Gibbs energy dissipation in heterotrophic growth exist, it ought to be possible to extend these for mixotrophic and autotrophic growth as well. It is the aim of this paper to develop a simple model for all these cases and to test it using published data [13-15] on mixotrophic and autotrophic growth. The thermodynamic characteristics of these metabolic processes will be compared with other data on chemo-heterotrophic growth and the endothermic nature of photosynthesis will be contrasted with the very special case of endothermic bacterial growth of $M$. barkeri.

\section{Simple stoichiometric model for chemotrophic, mixotrophic, and phototrophic growth}

Cellular growth can be pictured as a spontaneous transformation of a range of nutritional molecules such as glucose, amino acids, salts, etc., into new biomass, and possibly other products. Each type of growth reaction is characterized by its own stoichiometry and heat of reaction. All of these heat effects may be analyzed in a common framework by extending a simple stoichiometric model used in the literature so far for describing chemoheterotrophic growth [8] to mixotrophic and photoautotrophic growth.

The simple model for chemo-heterotrophic growth

This simple model for chemo-heterotrophic growth links the enthalpy and the Gibbs energy of reaction to the biomass yield $Y_{\mathrm{X} / \mathrm{S}}$ by subdividing the whole process into two partial reactions (Fig. 1). The reaction shown at the right of Fig. 1, called biosynthetic or anabolic reaction, describes the stoichiometry of the synthesis of new biomass from the chemicals that serve as sources for carbon, nitrogen, and other elements, whereas the catabolic or energy-yielding reaction at the left supplies the necessary energy by degrading the molecules used as energy sources such as glucose into lowenergy waste molecules such as carbon dioxide and water. The Gibbs energy that is released thereby in the form of ATP is consumed by biosynthesis and enables the latter to synthesize the energy-rich form of matter found in biomass from simple source molecules (Fig. 1).

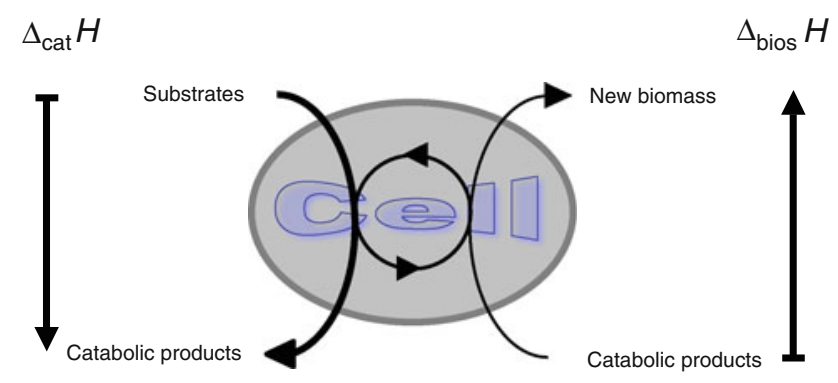

Fig. 1 Split of growth metabolism into an energy yielding and a biosynthetic reaction with, respectively, a negative and a positive enthalpy change. Adapted from [20]

The subdivision of the whole process into a catabolic and a biosynthetic reaction is often performed in a somewhat arbitrary way [16]. In this paper, for instance, it will be assumed that all of the nutritional substrates are first degraded into the products of catabolism (e.g., $\mathrm{CO}_{2}$ and $\mathrm{H}_{2} \mathrm{O}$ ) and that these are then partly transformed into biomass by the anabolic reaction. Although this represents a gross oversimplification, it affords particularly simple equations and will yield the correct results, as long as the formulation of biosynthesis is not used by itself without the energy yielding reaction [16].

In the way of an example, the two formal partial reactions for heterotrophic growth of algae on glucose as a carbon and energy source resulting from this split would read in C-molar notation:

Catabolism:

$\mathrm{CH}_{2} \mathrm{O}+\mathrm{O}_{2} \Longrightarrow \mathrm{CO}_{2}+\mathrm{H}_{2} \mathrm{O}$

Biosynthesis:

$$
\begin{aligned}
& \mathrm{CO}_{2}+0.93 \mathrm{H}_{2} \mathrm{O}+0.09 \mathrm{NO}_{3}^{-} \\
& \Longrightarrow \mathrm{CH}_{1.76} \mathrm{O}_{0.35} \mathrm{~N}_{0.09}+1.38 \mathrm{O}_{2}+0.09 \mathrm{OH}^{-}
\end{aligned}
$$

where $\mathrm{CH}_{1.76} \mathrm{O}_{0.35} \mathrm{~N}_{0.09}$ denotes the elemental chemical formula of one $\mathrm{C}$-mole of dry biomass without the ashes [14].

The stoichiometry of the whole growth reaction is obtained by multiplying Eq. 2 by $Y_{\mathrm{X} / \mathrm{S}}$ and adding Eq. 1 . The enthalpy of the whole growth reaction can therefore be linked to the biomass yield $Y_{\mathrm{X} / \mathrm{S}}$ as follows:

$\Delta_{\mathrm{r}} H=\Delta H_{\text {cat }}+Y_{\mathrm{X} / \mathrm{S}} \cdot \Delta H_{\text {bios }}$

where $\Delta_{\mathrm{r}} H, \Delta H_{\text {cat }}$, and $\Delta H_{\text {bios }}$ represent, respectively, the enthalpy of the whole growth reaction in kJ per C-mol of energy substrate, the enthalpy of catabolism and of anabolism. $Y_{\mathrm{X} / \mathrm{S}}$ stands for the biomass yield in C-mol of dry biomass formed per C-mol of energy substrate. An analogous equation may also be written for Gibbs energy:

$\Delta_{\mathrm{r}} G=\Delta G_{\text {cat }}+Y_{\mathrm{X} / \mathrm{S}} \cdot \Delta G_{\text {bios }}$ 
It is obvious from Fig. 1 and from Eq. 1 and 2 that the energy yielding reaction will be highly exothermic and exergonic, whereas the biosynthetic reaction will be endothermic and endergonic. Equation 3 predicts that both the heat and the Gibbs energy dissipation $\left(\Delta_{\mathrm{r}} H\right.$ and $\Delta_{\mathrm{r}} G$, respectively) will decrease, i.e., will have less negative values for higher biomass yields. This behavior has been confirmed for $\Delta_{\mathrm{r}} H$ in many calorimetric experiments [17] as shown for selected organisms in Fig. 4. Since $\Delta_{\mathrm{r}} G$ cannot become positive according to the second law of thermodynamics, Eq. $3 \mathrm{~b}$ may be used to predict the thermodynamically highest possible biomass yield by setting $\Delta_{\mathrm{r}} G$ to zero.

Extension to mixotrophic and photo-autotrophic growth

This simple picture may also be extended to describing mixotrophy by assuming that the flux of the energy yielding reaction will change as shown in Fig. 2 as one moves toward photosynthetic autotrophy. Figure 2 a shows the completely heterotrophic case with elements for the stoichiometry of aerobic respirative metabolism. In mixotrophic growth (Fig. 2b), the external energy source glucose will in part be replaced by photosynthate, which is synthesized from $\mathrm{CO}_{2}$ by using the energy from photons. The chemical reaction generating the photosynthate is the reverse of Eq. 1. The C-molar composition of photosynthate is assumed to be $\mathrm{CH}_{2} \mathrm{O}$, just as glucose. The consumption of external glucose thus decreases for the same amount of fresh biomass formed (Fig. 2b), so that the biomass yield based on glucose formally increases. In completely autotrophic growth, no glucose is consumed any more and $Y_{\mathrm{X} / \mathrm{S}}$ tends to infinity (Fig. 2c).

As the photosynthate is formed according to the reverse of Eq. 1, and because it may be assumed to be oxidized back to carbon dioxide in the energy yielding reaction (Eq. 1) of the photosynthetic cells, its formation and its oxidation cancel. The net effect of moving toward photosynthetic autotrophy will thus be nothing more than to reduce the exterior apparent consumption of the energy substrate, resulting in a formal increase of the biomass yield. The relationship between $\Delta_{\mathrm{r}} H$ and $Y_{\mathrm{X} / \mathrm{S}}$ (Eq. 3) remains valid, but the biomass yield increases as the system moves to photo-autotrophy. Equation $3 \mathrm{a}$ predicts that at a high enough biomass yield, the enthalpy of the growth reaction will exceed zero because the large positive term of biosynthesis will outweigh the negative catabolic enthalpy change in this equation. At that point, mixotrophic growth must become endothermic. By the same token, the Gibbs energy of the growth reaction (Eq. 3b) will also become positive. The second law of thermodynamics permits this now because there is an additional Gibbs energy source in the form of photons. In pure photoautotrophic growth, both $\Delta_{\mathrm{r}} G$ and $\Delta_{\mathrm{r}} H$ will tend to infinity because no energy substrate is consumed any more.

It is the aim of this contribution to demonstrate this transition toward endothermic growth experimentally.

\section{Experimental methods}

The green microalga $C$. vulgaris CCAP 211/11B was grown in a BioRC1. This was an isothermal reaction calorimeter based on the commercial RC1 from MettlerToledo AG (Switzerland) but modified for biological cultures. The BioRC1 consisted of a 2-L culture vessel that could be sterilized and was equipped with a gas sparger, a six blade turbine, baffles, as well as with all the usual paraphernalia needed to control or to monitor $\mathrm{pH}$, $\mathrm{pO}_{2}$, oxygen transfer rate, and carbon dioxide evolution rate. The reactor temperature was controlled at $(25.0000 \pm$ $0.0005){ }^{\circ} \mathrm{C}$ by automatically adapting the temperature of the cooling oil circulating at high rates in the cooling jacket. The heat dissipation rate was calculated on-line from the temperature difference between the reactor and the jacket, using a value of the heat transfer coefficient that was regularly measured by activating a calibration heater for brief periods of time. An exact description of this set-up has been published by Janssen et al. [13].

Light was delivered to the reactor either by two panels of 1,452 light emitting diodes (LED) installed close to the culture vessel $[13,15]$ or diffused directly into the culture broth by a quartz rod with a rough surface fitted to the head-plate of the BioRC1, and connected to a $150 \mathrm{~W}$ xenon lamp by a liquid light guide [14]. Alternatively, a fluorescent flat lamp was used in the latter set-up instead of the xenon light source. Appropriate measures were taken to avoid any thermal influence of the light sources on the
Fig. 2 Change of energy substrate (e.g. glucose) consumption flux when going from chemo-heterotrophic (a) to mixotrophic (b) and to photo-autotrophic growth (c)

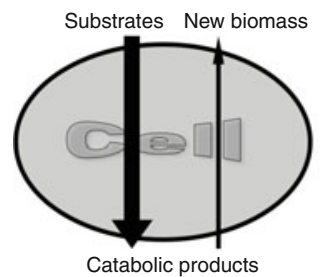

(a)

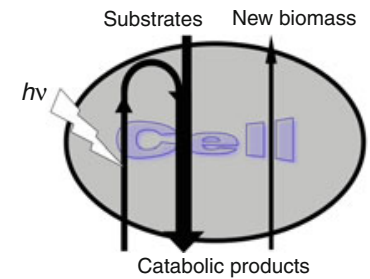

(b)

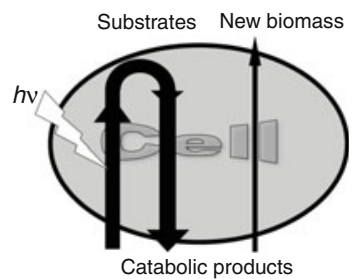

(c) 
culture medium as much as possible. Further details may be found in earlier publications [13-15].

\section{Results}

Photosynthetic growth per se is endothermic

The experiments done by Janssen et al. [13] demonstrate nicely the endothermic nature of photo-autotrophic growth. The experiment shown in Fig. 3 was performed with the LED light sources around the biophotocalorimeter. C. vulgaris was grown in it in a completely autotrophic mode. After reactor inoculation, the calibration heater was turned on briefly in order to measure the heat transmission coefficient and then the lights were turned on ("Lights on"). The immediate large increase in the heat power signal $\mathrm{P}$ indicated the light intensity that was absorbed by the reactor hardware and by the still small amount of biomass present. From $t=20 \mathrm{~h}$ onwards, the heat dissipation signal increased in parallel with the biomass density (not shown). After $50 \mathrm{~h}$, the power reached a maximum, indicating that from this moment onwards all the light was absorbed by the biomass and partly dissipated as heat. During the following period growth was limited by the influx of light and the OD increased only linearly (not shown). The dissolved oxygen concentration increased to almost $104 \%$ saturation, thus demonstrating the oxygen generation by photosynthesis (data not shown).

At $t=74 \mathrm{~h}$, the activity of photosystems I and II were blocked by adding two inhibitors (DBMIB and DCMU, respectively). The dissolved oxygen dropped immediately to $95 \%$ saturation, reflecting the end of oxygen generation (not shown). As shown in Fig. 3, the heat dissipation rate jumped up by an amount of $62.5 \mathrm{~mW} \mathrm{~L}^{-1}$ to a higher level, indicating that after the inhibition all the light shone into the reactor was dissipated as heat. The fact that the heat dissipation was lower by $62.5 \mathrm{~mW} \mathrm{~L}^{-1}$ when photosynthesis was going on demonstrates clearly that

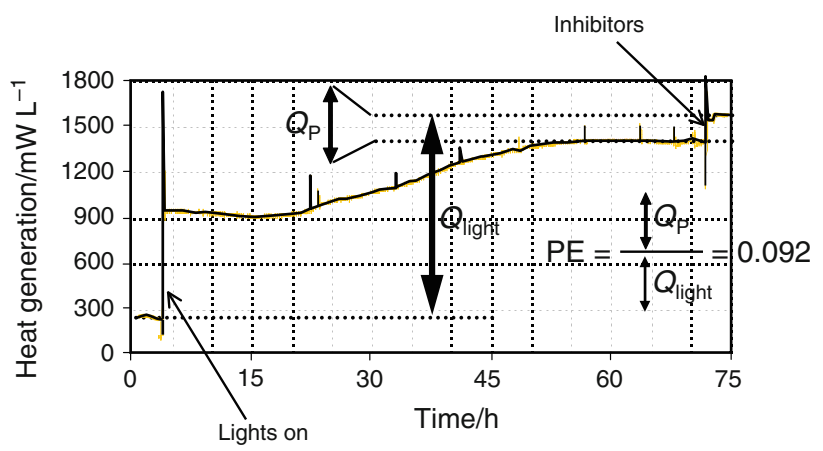

Fig. 3 Measured heat generation rate in purely phototrophic growth of C. vulgaris. Adapted from [13] photosynthetic growth is intrinsically endothermic by this amount. This does not mean that such experiments are endothermic as a whole because the absorption of large amounts of excess light will always generate a lot of heat.

Similar experiments with Chlorella sorokiniana at a temperature of $37^{\circ} \mathrm{C}$ confirmed these observations [15]. Improved growth conditions and calorimetric measuring techniques permitted measuring the endothermic heat of growth not only once at the end of the culture, but to monitor it continuously during most of the experiments.

The transition from heterotrophic to mixotrophic to autotrophic growth

The algal strain $C$. vulgaris was grown in a number of batch and fed-bach experiments in the RC1-based biophotocalorimeter (for details see [14]). Light was fed from the external xenon lamp into the culture medium by means of a liquid light guide. By adding various amounts of glucose and turning the lamp on or off the cells could be grown either heterotrophically or in various mixotrophic modes. Autotrophic growth was explored after a considerable amount of biomass had formed in mixotrophic growth and once glucose was exhausted. Growth was quantified by systematic sampling and off-line analysis, and the calorimeter provided an on-line reading of the heat dissipation rate. The heat yields, or enthalpies of growth, were obtained by integrating this signal over a given period of time and by comparing it with the increase in dry biomass. The resulting values are plotted against the biomass yield $Y_{\mathrm{X} / \mathrm{S}}$ in C-mol of biomass grown per C-mol of glucose consumed in Fig. 4.

For completely autotrophic growth with $Y_{\mathrm{X} / \mathrm{S}}=\infty$, the data obtained from [14] was completed by results from another experiment [15]. Since in this study both the heat dissipation rate and the rate of $\mathrm{CO}_{2}$ assimilation were monitored on-line, the heat of growth $\Delta_{\mathrm{r}} H_{\mathrm{X}}$ could be evaluated continuously during the whole duration of the culture simply by dividing the former signal through the latter. The values fell into a range indicated by the half transparent square overlying the two black dots.

The lines in Fig. 4 represent predictions for the enthalpies of growth. They were computed by using Eq. 4a, which was derived by dividing Eq. 3a by the biomass yield in order to avoid infinite $\Delta_{\mathrm{r}} H$ values resulting from infinite biomass yields when no glucose is consumed in autotrophic growth:

$$
\begin{aligned}
& \Delta_{\mathrm{r}} H_{\mathrm{X}}=\frac{1}{Y_{\mathrm{X} / \mathrm{S}}} \cdot \Delta H_{\mathrm{cat}}+\Delta H_{\mathrm{bios}} \\
& \Delta_{\mathrm{r}} G_{\mathrm{X}}=\frac{1}{Y_{\mathrm{X} / \mathrm{S}}} \cdot \Delta G_{\mathrm{cat}}+\Delta G_{\mathrm{bios}}
\end{aligned}
$$




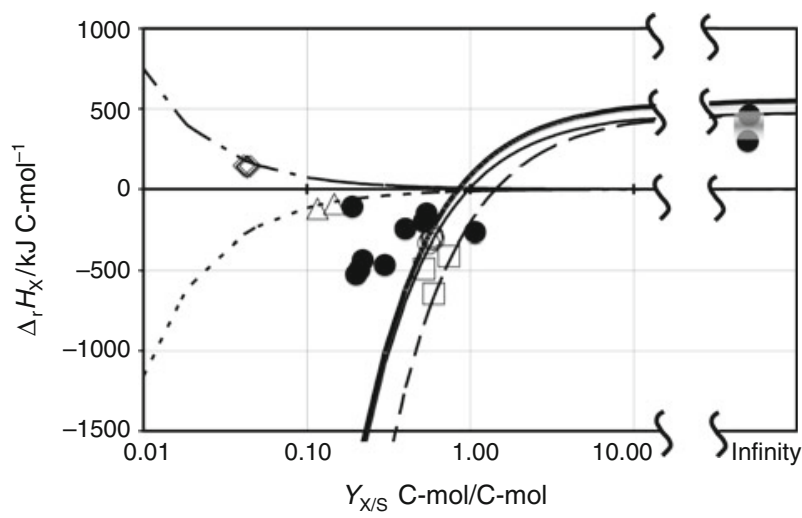

Fig. 4 Calorimetrically measured (markers) and calculated enthalpy changes $\Delta_{\mathrm{r}} H_{\mathrm{X}}$ (lines) for various microbial and algal growth systems. Solid dots and thick line heterotrophic, mixotrophic and autotrophic growth of C. vulgaris [14], half transparent square overlying two solid dots range of values observed in autotrophic growth of C. sorokiniana [15], open circles and thin solid line aerobic growth on glucose by several yeasts and E. coli [8], open squares and broken line aerobic growth of yeast on ethanol [8], open triangles and dotted line anaerobic fermentative growth of two different yeasts on glucose producing ethanol [8], open diamonds and dash-dotted line acetotrophic methanogenesis of $M$. barkeri [9]

where $\Delta_{\mathrm{r}} H_{\mathrm{X}}$ and $\Delta_{\mathrm{r}} G_{\mathrm{X}}$ denote the enthalpy and the Gibbs energy per $\mathrm{C}$-mole of biomass grown, respectively.

The prediction of Eq. $4 \mathrm{a}$ for algae is represented in Fig. 4 by the thick solid line. It clearly predicts endothermic growth for biomass yields above a certain value close to unity. The solid dots reflect the experimental results, which confirm that growth becomes endothermic as it shifts toward autotrophy. Unfortunately these points are dispersed by large calorimetric measurement inaccuracies. If the energy balance had closed, the points would have to lie right on the thick line.

For comparison, similar data has been added for microbial growth published earlier [8, 9, 17]. The thin solid line on Fig. 4 represents the prediction for heterotrophic growth of aerobic microorganisms on glucose. It is somewhat different from the thick line because microorganisms use ammonium ions as their nitrogen source and not nitrate as algae. Also represented are yeasts aerobically growing on ethanol (broken line) and yeasts growing anaerobically on glucose (dashed line). A particularly interesting case is anaerobic acetotrophic methanogenesis (dash-dotted line). For this type of chemoheterotrophic fermentation, the graph predicts endothermic growth at small biomass yields [9]. Table 1 lists the values for $\Delta H_{\text {cat }}$ and $\Delta H_{\text {bios }}$ used for the construction of these lines.

The measured enthalpies of microbial growth (open symbols) fit the predictions much better than the ones for algae (solid symbols) and demonstrate that the measurement of heat effects in heterotrophic microbial growth is clearly much more accurate than in algae. This is even the case for the bizarre endothermic growth by acetotrophic methanogenesis. The study by Liu et al. [9] remains the only calorimetric demonstration of the existence of an endothermic non-photosynthetic life form. These bacteria will truly cool their environment down while growing. Although the curves of the aerobic microorganisms also extend into the endothermic region, they cannot be grown at biomass yields high enough to make the process endothermic because their chemo-heterotrophic nature forces them to oxidize large amounts of glucose to $\mathrm{CO}_{2}$ in order to generate the energy needed for growth.

\section{Interpretation based on a Gibbs energy analysis}

The general framework of a Gibbs energy analysis

The results presented in the previous chapter demonstrate that chemo-heterotrophic growth is virtually always strictly exothermic. Only one highly unusual fermentation reaction was ever found that actually absorbs heat [9]. On the other hand, cultures that grow mixotrophically may become endothermic if the photosynthetic part of the metabolism is important enough and photoautotrophic growth is definitely endothermic. These observations and the conditions for endothermic growth can be explained and analyzed based on a Gibbs energy analysis.

Table 1 Enthalpies of catabolic and biosynthetic reactions used for constructing lines in Fig. 4

\begin{tabular}{lccc}
\hline & $\Delta H_{\text {cat }} / \mathrm{kJ} \mathrm{C}^{-\mathrm{mol}^{-1}}$ & $\Delta H_{\text {bios }} / \mathrm{kJ} \mathrm{C}^{-\mathrm{mol}^{-1}}$ & Source $^{-1}$ \\
\hline Hetero-, mixo-, autotrophic growth & -467.8 & 555.0 & \\
Aerobic growth glucose (yeast) & -467.8 & 478.5 & {$[8,21]$} \\
Aerobic growth ethanol & -683.4 & 478.5 & {$[8]$} \\
Ethanol fermentation glucose & -11.6 & 0.3 & {$[8]$} \\
Acetotrophic methanogenesis & +7.5 & 0.4 & {$[8]$} \\
\hline
\end{tabular}

$\Delta H_{\text {bios }}$ for heterotrophic, mixotrophic and photoautotrophic growth was estimated from the heat of combustion of dry algal biomass of the elemental composition cited in Eq. 2 [14]. The degree of reduction is therefore $\gamma_{\mathrm{X}}^{\mathrm{O}}=5.06$, the heat of combustion may be estimated as 5.06 ( $109 \mathrm{~kJ} \mathrm{e} \mathrm{mol}^{-1}$ ) [22]. For obtaining $\Delta H_{\text {bios }}$, the negative heat of combustion must in addition be corrected with the enthalpy of formation of infinitely diluted nitrate [21] and for the nitrogen content of the dry biomass 


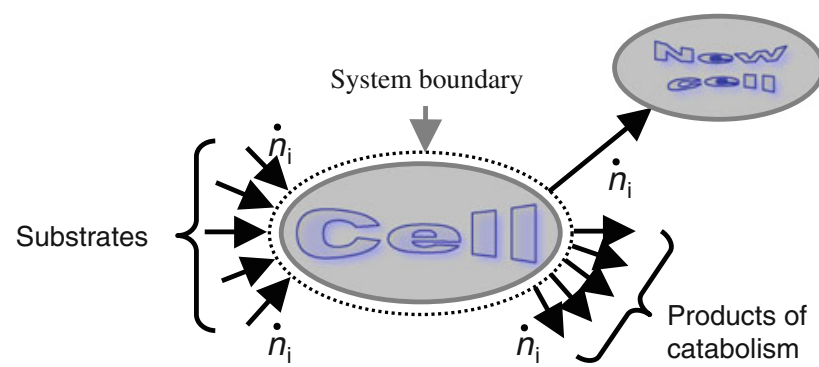

Fig. 5 Gibbs energy balance around a live cell. Adapted from [17]

Based on Fig. 5, a Gibbs energy balance around a living, growing cell may be formulated as follows:

$\frac{\mathrm{d} G}{\mathrm{~d} T}=0=\dot{W}+\sum_{i} \mu_{i} \cdot \dot{n}_{i}-T \cdot \dot{S}_{\mathrm{prod}}$

According to this balance, the Gibbs energy accumulation within the cell, represented by the left hand side of the equation, is given by the sum of all fluxes of Gibbs energy into and out of the cell, minus the rate of Gibbs energy destruction due to irreversible processes. The first right hand term denotes the influx due to work done to the cells, whereas the second term represents the sum of import and export of Gibbs energy by the exchange of metabolites with the environment. (Metabolites that are imported have a positive flux $\dot{n}_{i}$ whereas the flux of exported metabolites is negative.) The last term quantifies the rate of Gibbs energy dissipation and can be derived from a combination of an entropy and an energy balance [8].

If the cell is assumed to be in a steady state, the accumulation of Gibbs energy must be zero. It can also be shown [8] that in this case the second term on the right hand side may be replaced by the negative value of the Gibbs energy of the growth reaction $\Delta_{\mathrm{r}} G_{\mathrm{X}}$ times the rate of advancement of this reaction. Equation 5 thus becomes:

$\Delta_{\mathrm{r}} G_{\mathrm{X}} \cdot r_{\mathrm{X}} \cdot V=\dot{W}-T \cdot \dot{S}_{\text {prod }}$

\section{Chemo-heterotrophic growth}

The case of chemo-heterotrophic growth was analyzed in great detail [8, 16-19]. Such organisms do no work on their environment and $\dot{W}$ is zero. For this case, Equation 6 demonstrates that the Gibbs energy of the growth reaction must be negative, as the entropy generation rate can only be positive. The former then reflects directly the rate of entropy generation, which according to irreversible thermodynamics is the driving force for the process. The higher the entropy generation, i.e., the more negative $\Delta_{\mathrm{r}} G_{\mathrm{X}}$, the larger will the driving force be for metabolism and the faster growth will occur.
Several authors evaluated the Gibbs energy change of a large number of microbial growth reactions based on measured growth stoichiometries [18-20]. They found that for a large majority of microbial growth systems $\Delta_{\mathrm{r}} G_{\mathrm{X}}$

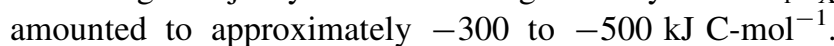
This was attributed to a compromise reached by evolution between high thermodynamic driving forces for growth on the one hand and high biomass yield on the other. The conflict between these two targets is exemplified by Eq. $4 \mathrm{~b}$. Organisms with an unusually negative $\Delta_{\mathrm{r}} G_{\mathrm{X}}$ have high metabolic driving forces and thus a fast metabolism. But according to Eq. 4b, they also suffer from a low biomass yield and may have been outgrown during evolution by more efficient competitors. Those with an unusually high $Y_{\mathrm{X} / \mathrm{S}}$, however, may have suffered from such low thermodynamic driving forces that they grew much too slowly to survive in nature. This may have been the reason for emergence of a "reasonable" average value of $\Delta_{\mathrm{r}} G_{\mathrm{X}}$.

The Gibbs energy of the growth reaction is linked to the heat of growth by the following equation:

$\Delta_{\mathrm{r}} G_{\mathrm{X}}=\Delta_{\mathrm{r}} H_{\mathrm{X}}-T \Delta_{\mathrm{r}} S_{\mathrm{X}}$

where $\Delta_{\mathrm{r}} S_{\mathrm{X}}$ denotes the entropy change between the products and the substrates of the growth reaction. In aerobic growth, this term is so small that the heat generation $\Delta_{\mathrm{r}} H_{\mathrm{X}}$ is comparable in size to $\Delta_{\mathrm{r}} G_{\mathrm{X}}$ [8], thus explaining why all aerobic life processes dissipate an amount of heat in the order of -300 to $-500 \mathrm{~kJ} \mathrm{C} \mathrm{mol}^{-1}$.

The situation is different for organisms with a fermentative catabolism. Since no external electron acceptor is involved, the enthalpy change $\Delta_{\mathrm{r}} H_{\mathrm{X}}$ may be quite small. On the other hand, these energy-yielding reactions rip the molecules serving as energy sources apart into smaller pieces, which thus accounts for a large positive $T \Delta_{\mathrm{r}} S_{\mathrm{X}}$ term. In these cases, it is this large $T \Delta_{\mathrm{r}} S_{\mathrm{X}}$ that makes $\Delta_{\mathrm{r}} G_{\mathrm{X}}$ negative [8]. In the endothermic growth based on acetotrophic methanogenesis explored by Liu et al. [9], this entropy increase is so large that the bacteria can grow despite of the fact that they are forced to assimilate a substrate with less energy content than the waste products and to take the difference up in the form of heat. This explains why acetotrophic methanogenesis is an endothermic process.

\section{Photoautotrophic and mixotrophic growth}

In the case of partially or wholly photosynthetic growth, photons supply some of the energy for metabolism and must be accounted for by a positive term $\dot{W}$ in the Gibbs energy balance Eq. 6 . While the irreversible entropy generation still represents the intrinsic thermodynamic driving force for the growth process, $\Delta_{\mathrm{r}} G_{\mathrm{X}}$ is now uncoupled from 


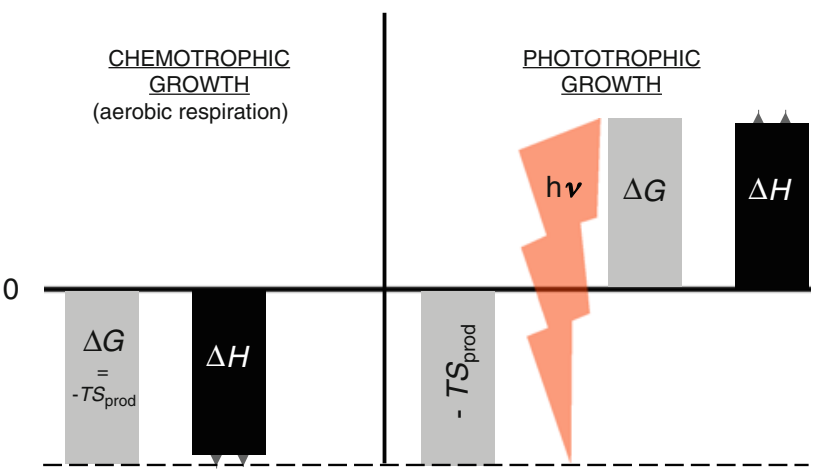

Fig. 6 Qualitative comparison of energy exchange between chemoheterotrophic and photo-autotrophic growth. In the former $\Delta_{\mathrm{r}} G_{\mathrm{X}}$ must be negative because it reflects directly the negative entropy production times $T$, whereas in the latter case $\Delta_{\mathrm{r}} G_{\mathrm{X}}$ is uncoupled from $T \cdot S_{\text {prod }}$, because the photons are an additional source of Gibbs energy

it and may adopt a more positive or even a downright positive value.

A comparison between the energy exchanges in aerobic heterotrophic and photoautotrophic growth is given in Fig. 6. The bar at the left shows the amount of Gibbs energy that must be dissipated in heterotrophic growth for providing the necessary driving force. The next bar to the right schematically depicts $\Delta_{\mathrm{r}} H_{\mathrm{X}}$ for aerobic growth, which is practically as negative as $\Delta_{\mathrm{r}} G_{\mathrm{X}}$ because $T \Delta_{\mathrm{r}} S_{\mathrm{X}}$ is close to zero (small grey triangles at the bottom of $\Delta_{\mathrm{r}} H_{\mathrm{X}}$ ). The height of the lightning arrow to the right of the dividing line represents the energy that could in principle be harnessed from photons in photosynthetic growth processes. Some of this energy must necessarily be dissipated in order to provide the necessary driving force. It is represented by the grey bar labeled $-T \cdot S_{\text {prod. }}$ But another part can now be recovered in the chemical potential of the synthesized biomass, thus making $\Delta_{\mathrm{r}} G_{\mathrm{X}}$ positive. The stoichiometry of this synthesis process is given by Eq. 2 and is characterized by a similar small reaction entropy $\left(\Delta_{\mathrm{r}} S_{\mathrm{X}}\right)$ as heterotrophic growth. The enthalpy change for this process must therefore be similar to $\Delta_{\mathrm{r}} G_{\mathrm{X}}$ and is thus also positive, making this an endothermic reaction.

It could be speculated that the true driving force for photoautotrophic growth, the entropy generation times $T$, should be on the same order of magnitude of $-500 \mathrm{~kJ}$ $\mathrm{C}-\mathrm{mol}^{-1}$ as was found for many chemo-heterotrophic processes. Figure 7 reports approximate values for the measured energy exchange in photoautotrophic growth of C. vulgaris. The light available to the culture was measured directly by calorimetry and accounted for roughly $5000 \mathrm{~kJ} \mathrm{C}-\mathrm{mol}^{-1}$ of new dry biomass grown. $\Delta_{\mathrm{r}} G_{\mathrm{X}}$ and $\Delta_{\mathrm{r}} H_{\mathrm{X}}$ were evaluated from tables as +482.4

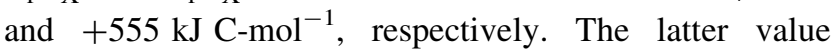

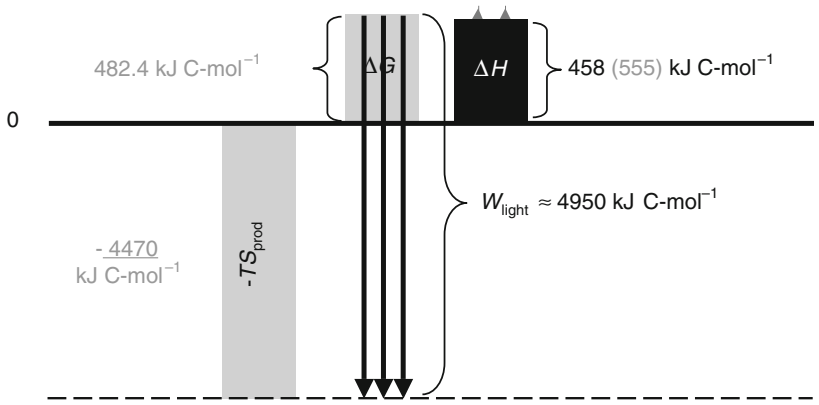

Fig. 7 Estimation of Gibbs energy dissipation in photosynthesis based on the data of Janssen et al. [12]. Black surfaces and black font figures measured values, grey surfaces and figures calculated values

corresponded quite well to the calorimetric measurement,

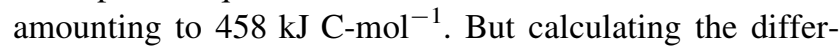
ence between the source energy in the form of light and the amount of energy recovered in the biomass yielded almost

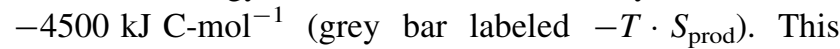
corresponds to an amount of dissipated energy, i.e, a thermodynamic driving force that was practically an order of magnitude higher than what would be expected for chemo-heterotrophic growth.

A higher Gibbs energy dissipation in photoautotrophic growth as compared to chemoheterotrophic growth would however be expected because the former involves many more biochemical processes, and thus needs a higher driving force. It is commonly accepted that 12 photons are required for an efficient reduction of one mole of $\mathrm{CO}_{2}$ to photosynthate [14]. As the light used in the experiments described here had a wave length of $665 \mathrm{~nm}$, this represents an energy input of $2081 \mathrm{~kJ} / \mathrm{C}-\mathrm{mol}$. Substracting from this the $\Delta_{\mathrm{r}} G_{\mathrm{X}}$ of $+482.4 \mathrm{~kJ}$ that is stored in one C-mole of photosynthate leaves us with a Gibbs energy dissipation of $1600 \mathrm{~kJ} / \mathrm{C}-\mathrm{mol}$ for this part of the metabolism alone. Since the photosynthate is subsequently used for biomass synthesis in a process that is analogous to chemoheterotrophic growth, a further Gibbs energy dissipation in the order of $500 \mathrm{~kJ} / \mathrm{C} / \mathrm{mol}$ may be expected to serve as driving force for this part of the metabolism. As a result, over $2000 \mathrm{~kJ} / \mathrm{C}-\mathrm{mol}$ out of the observed dissipation of $4500 \mathrm{~kJ} /$ $\mathrm{C}$-mol might be required as metabolic driving force.

It is obvious that much of the remaining unaccounted energy dissipation must have been absorbed by the culture or the bioreactor components uselessly and dissipated without having contributed anything to drive metabolism. Although it might be a formidable challenge to measure only the energy dissipation that is "useful" as a driving force for the cells, there is still a lot of room for improving experimental techniques in calorimetry, and it is hoped that such improvements might sooner or later at least yield an improved approximation of this value. 


\section{Conclusions}

Calorimetry is a convenient method for exploring quantitatively photosynthetic growth. In addition to yielding data on the heat effects of photosynthetic processes it offers an unusually easy possibility to measure the total light intensity absorbed by the culture. The results discussed in this contribution reconfirm that photosynthesis is an endothermic process and yield approximate values for the absorbed heat.

Heat effects in photoautotrophic and in mixotrophic growth may be predicted on the basis of the biomass yield using the same simple stoichiometric calculation as the one used for chemo-heterotrophic growth. This was qualitatively confirmed by growing the algal strain $C$. vulgaris in a reaction calorimeter hererotrophically, mixotrophically and autotrophically. Unfortunately, the comparison for mixotrophic experiments revealed large measurement inaccuracies and the need for improved calorimetric measurement techniques. The autotrophic experiments with $C$. vulgaris and $C$. sorokiniana were in fair agreement with the calculations. They were used to estimate the Gibbs energy dissipation, which may be regarded as the overall driving force for the process. It turned out to be an order of magnitude higher that typical values for chemotrophic growth.

Almost half of this dissipation may be shown to be biochemically needed as a thermodynamic driving force for photosynthetic growth. How much of the remaining Gibbs energy dissipation is also acting as a driving force for metabolism as opposed to being physically absorbed and turned into heat remains to be explored using a considerably improved calorimetric measuring technique.

Acknowledgements The authors gratefully acknowledge important funding for this work by the Swiss National Science Foundation (SNF).

\section{References}

1. Cooney CL, Wang DIC, Mateles RI. Measurement of heat evolution and correlation with oxygen consumption during microbial growth. Biotechnol Bioeng. 1968;11:269-81.

2. Luong JHT, Volesky B. A new technique for continuous measurement of the heat of fermentation. Eur J Appl Microbiol Biotechnol. 1982;16:28.

3. Birou B, Marison IW, von Stockar U. The calorimetric investigation of aerobic fermentations. Biotechnol Bioeng. 1987;30: $650-60$.
4. Randolph TW, Marison IW, Berney C, von Stockar U. Bench scale calorimetry of hybridomas in suspension culture. Biotechnol Tech. 1989;3:369-74.

5. Marison IW, von Stockar U. Large-scale calorimetry and biotechnology. Thermochim Acta. 1991;193:215-42.

6. Kemp RB, Guan YH. The application of heat flux measurement to improve the growth of mammalian cells in culture. Thermochim Acta. 2000;332:23-30.

7. Maskow T, Babel W. Calorimetric investigation of bacterial growth on phenol. Thermochim Acta. 1998;309:97-103.

8. von Stockar U, Liu JS. Does microbial life always feed on negative entropy? Thermodynamic analysis of microbial growth. Biochim Biophys Acta. 1999;1412:191-211.

9. Liu JS, Marison W, von Stockar U. Microbial growth by a net heat up-take: a calorimetric and thermodynamic study on Acetotrophic Methanogenesis by Methanosarcina barkeri. Biotechnol Bioeng. 2001;75:170-80.

10. Magee JL, DeWitt TW, Coolidge E, Smith F, Daniels F. A photocalorimeter. The quantum efficiency of photosynthesis in algae. J Am Soc. 1939;61:3529-33.

11. Johansson P, Wadsö I. A photo microcalorimetric system for studies in plant tissue. J Biochem Biophys Methods. 1997;35: 103-14.

12. Battley EH. Studies on anaerobic growth of a biotype of Chlorella vulgaris. Antonio van Leeuwenhoek. 1968;30:81-96.

13. Janssen M, Patiño R, von Stockar U. Application of bench-scale biocalorimetry to photoautotrophic cultures. Thermochim Acta. 2005;435:18-27.

14. Patiño R, Janssen M, von Stockar U. A study of the growth for the microalga Chlorella vulgaris by photo-bio-calorimetry and other on-line and off-line techniques. Biotechnol Bioeng. 2007; 96:757-67.

15. Janssen M, Wijffels R, von Stockar U. Biocalorimetric monitoring of photoautotrophic batch cultures. Thermochim Acta. 2007;458:54-64.

16. von Stockar U, Vojinovic V, Maskow T, Liu JS. Can microbial growth yield be estimated using simple thermodynamic analogies to technical processes? Chem Eng Process. 2008;47:980-90.

17. von Stockar U, Maskow T, Liu JS, Marison WI, Patiño R. Thermodynamics of microbial growth and metabolism: an analysis of the current situation. J Biotechnol. 2006;121:517-33.

18. Heijnen JJ, van Dijken JP. In search of thermodynamic description of biomass yields for the chemotrophic growth of microorganisms. Biotechnol Bioeng. 1992;39:833-58.

19. Heijnen JJ, van Dijken JP. Response to comments on: in search of a thermodynamic description of biomass yields for the chemotrophic growth of micro-organisms. Biotechnol Bioeng. 1993;42: 1127-30.

20. Liu JS, Vojinovic V, Patiño R, Maskow T, von Stockar U. A comparison of various Gibbs energy dissipation correlations for predicting microbial growth yields. Thermochim Acta. 2007; 458:38-46.

21. Roels JA. Energetics and kinetics in biotechnology. Amsterdam: Elsevier Biomedical Press; 1983.

22. von Stockar U. Biothermodynamics of live cells. A tool for biochemical engineering. J Non-Equilib Thermodyn. 2010;35: $415-75$. 